\title{
REPORT ON RESEARCH WITH BIOMIMETIC AUTONOMOUS UNDERWATER VEHICLE - NAVIGATION AND AUTONOMOUS OPERATION
}

\author{
Tomasz Praczyk*, Piotr Szymak**, Krzysztof Naus*, \\ Leszek Pietrukaniec*, Stanisław Hożyń** \\ * Polish Naval Academy, Faculty of Navigation and Naval Weapons, Śmidowicza 69 Str., 81-127 \\ Gdynia, Poland; e-mail: \{t.praczyk; k.naus; l.pietrukaniec\}@amw.gdynia.pl \\ ** Polish Naval Academy, Faculty of Mechanical and Electrical Engineering, Śmidowicza 69 Str., 81-127 \\ Gdynia, Poland; e-mail: \{p.szymak; s.hozyn\}@amw.gdynia.pl
}

\begin{abstract}
The paper presents the second part of the final report on all the experiments with biomimetic autonomous underwater vehicle (BAUV) performed within the confines of the project entitled 'Autonomous underwater vehicles with silent undulating propulsion for underwater ISR', financed by Polish National Center of Research and Development. The report includes experiments on the swimming pool as well as in real conditions, that is, both in a lake and in the sea. The tests presented in this part of the final report were focused on navigation and autonomous operation.
\end{abstract}

\section{Key words:}

autonomous underwater vehicle (AUV), biomimetic autonomous underwater vehicle (BAUV), course and depth control, autonomous control.

Research article

(C) 2018 Tomasz Praczyk, Piotr Szymak, Krzysztof Naus, Leszek Pietrukaniec, Stanisław Hożyń This is an open access article licensed under the Creative Commons Attribution-NonCommercial-NoDerivatives 4.0 license (http://creativecommons.org/licenses/by-nc-nd/4.0/) 


\section{INTRODUCTION}

Autonomous underwater vehicles (AUVs), as the name implies, are vehicles which need the ability to operate autonomously, or in other words, independently of a human being. This ability can be used on different levels and in different situations, e.g. during the entire operation of the vehicle - a vehicle completely independent of an operator, or only when a communication system with the operator is broken a remotely operated vehicle with a function to safely come back to the operator. To act autonomously, not only in emergency situations, each AUV has to be able to perform many different tasks, among other things: to estimate its position, to follow a predefined path, to detect obstacles, to build a map of the environment, to avoid collisions, to record data from the sensors, to receive commands and send data from/to the operator.

The AUV with the abilities outlined above was constructed within the framework of the project No. DOBR-BI04/033/13015/2013, entitled 'Autonomous underwater vehicles with silent undulating propulsion for underwater reconnaissance' financed by National Center of Research and Development [7] (see fig. 1). All the effort necessary to build the AUV, which due to a modern undulating propulsion was called biomimetic AUV, or in short BAUV $[1,7,8]$, was divided into many stages. First, conceptual works were done focused on both the hardware and the software $[2,4]$. Simultaneously, all external devices and sensors of the future BAUV were tested in separation in order to acquire practical knowledge concerning their application and their actual parameters and properties. The selection of the devices and sensors to include into the BAUV was dictated by vehicle tasks defined in assumptions to the project. In effect of the conceptual works initial designs of hardware and software were proposed. They enabled implementation of some software components and simulation tests whose goal was to lead the software to the state permitting its safe application on ready hardware [5, 6]. Simultaneously with simulation tests, hardware part of the BAUV was gradually constructed. Once both components were ready to combine, the experiments with complete vehicle were possible. The essential information about sensors and construction was presented in the first part of the report entitled: 'Report on research with biomimetic autonomous underwater vehicle - low level control'.

The present paper describes the second stage of the experiments, which were conducted in order to elaborate navigation system and autonomous operation. The remaining part of the paper is organized as follows: section 2 gives objectives 
of all the experiments, section 3 details individual parts of the experiments, and the final section summarizes the paper.

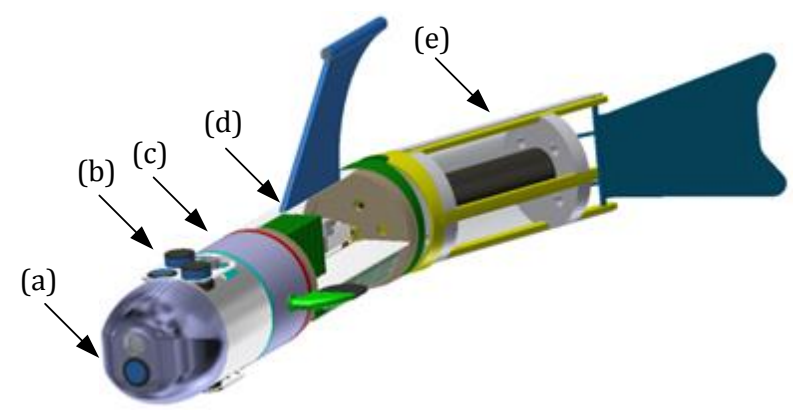

Fig. 1. 3D model of the BAUV, the vehicle consists of the following modules:

(a) module with camera and 'looking' forward echosounder, (b) sensor module with USBL, hydromodem, 'looking' up echosounder, sonar and 'looking' down echosounder, (c) module

of the lateral fins, (d) module of electronics and batteries, (e) module with the tail fin

\section{OBJECTIVES OF EXPERIMENTS}

The experiments with the real BAUV had the following objectives:

1. Verification of BAUV responses to emergency situations. In order to ensure safety of BAUV operation, the vehicle was equipped with software components responsible for detection of the emergency situations and activation appropriate responses to them. Application of the BAUV in the sea conditions required detailed tests of the mentioned components.

2. Testing navigational system (NS). The key vehicle ability, required for path following task is the ability to fix position. The position can be determined by GPS, USBL or be calculated by means of dead reckoning based on vehicle speed, course and depth. In order to test operation of NS and accuracy of position fixed by the system, series of experiments were necessary.

3. Testing ability of the vehicle to move along a desired path. The primary task of the BAUV is to move along a desired path and to perform additional operations on the way, e.g. operations related to cameras and sonar. In order to verify ability of the BAUV to move along a predefined path, series of experiments were necessary.

4. Testing ability to avoid collisions. The key skill of the BAUV is to detect obstacles, to build a map of the environment, and to avoid collisions. In order to verify ability of the BAUV to perform all the above tasks, separate tests were necessary. 
5. Comprehensive testing of BAUV ability to perform a mission. As mentioned above, the primary task of the BAUV is to move along a desired path and simultaneously to perform additional operations on the way, mainly operations related to recording data from cameras and sonar. The task above can be performed in autonomous mode and in that case we say that the BAUV performs a mission. In order to verify ability of the vehicle to perform a complete mission consisting of different operations, the final tests were required.

All the above mentioned tests took place in five various water areas. The short description of environments, which were utilized for the purpose of conducting the experiments, was presented in the article entitled: Report on research with biometric autonomous underwater vehicle - low level control.

\section{EXPERIMENTS}

\section{Tests on emergency situations}

The tests with emergency situations were a preparation of the BAUV for occurrence of different critical situations which can happen during mission or remote control. The examples of such situations are: low battery level, a key process missing in the system, a key device or sensor, e.g. compass, is unavailable or it does not work, obstacles cannot be detected - breakdown of a front echo-sounder, water detected inside the hull. In order to test a respond of the vehicle to all identified emergency situations, it was examined both in stationary conditions, that is, without launching, for example, by simulation of 'death' of a process, and then, in the pool, also by simulation of a critical event.

According to the results, time parameters which determined speed of response for a definite situation needed improvement. For example, it was necessary to prolong a permissible duration of vehicle 'relax' state that is the state in which the vehicle is not in the course of mission and it also does not perform any operation in the remotely operated mode. The occurrence of that state is interpreted as an error in communication between the CCC and the BAUV and in that case the vehicle surfaces and transmits its position.

\section{Tests on navigational system}

Experiments with the NS started in Polish Naval Academy with tests on GPS and vehicle ability to receive and to properly interpret position generated by USBL. Moreover, speed of GPS position recovery after its loss was tested. 
The initial tests showed that USBL position is received correctly. The GPS position after losing signal is recovered, practically, almost immediately which means no delays for cyclic GPS position recording to reduce a dead reckoning position error.

Next tests were focused on the remaining devices and sensors for navigation, that is, on compass OS5000, inertial navigational system INS-VN200, and on pressure sensor. The primary parameter when testing compass and INS was vehicle course. There was examined stability of course and correctness of indications. Generally, it appeared that course taken from the compass is more stable and accurate. A building in Polish Naval Academy of known orientation was used as an orientation pattern. Tests with each device were always proceeded by their calibration. After selecting OS5000 compass as a main source of information about the course, insignificant filtration of its outputs was necessary, filtrated signal was nothing but an average value determined in a short time window. This way, more stable course was achieved, which, in turn, contributed to improvement of LLCS (Low Level Control System) performance. More stable course implied more stable vehicle behaviour, changes in control mode were rare after averaging signal from compass.

VN200 was applied as a source of information about the remaining spatial orientation parameters. Echo-sounders and collision avoidance system were the main receivers of the information from VN200. They need information about pitch and roll angles to determine distance to obstacle or the sea bottom.

With regard to the pressure sensor, calibration was carried out whose objective was to determine a pressure corresponding to 'zero' depth of the vehicle. The 'zero' depth is the depth for which the vehicle is submerged up to the half of its upper fin. The fin is motionless and it houses all vehicle antennas, e.g. GPS, radiomodem antennas. The 'zero' depth means that all the antennas are situated above the surface and in consequence they are operable. Moreover, it also corresponds to 'relax' state of the vehicle in which its propellers does not work. In order to achieve the 'zero' depth, appropriate balancing was necessary which was accomplished through locating weights in the vehicle keel. Their location in the keel was adjusted to achieve a zero trim. The 'zero' depth is also the consequence of positive buoyancy of the vehicle which is both advantageous and disadvantageous. The advantage of that solution is that the vehicle will go up to the surface, it will not sunk, in case of any emergency situation, for example when propellers stop working. In turn, the disadvantage is that the maintenance of the depth always requires operation of lateral fins, the vehicle cannot stop underwater and stay motionless.

Extra tests devoted to the vehicle navigation were focused on determining its progressive and turning velocity for operating depth and course controllers and 
they were performed on the indoor pool. Determining those velocities was necessary to apply the dead reckoning when there is no a speedometer.

In order to fix the progressive velocity, the vehicle was covering the distance 20 meters a number of times to calculate an average velocity. This velocity was calculated for all assumed vehicle working modes in which parameters does not change value. The average turning velocity was calculated in the same way, the only difference was turn of the vehicle by 180 degrees. Next tests of the NS were focused on the following path task so they are described in the subsequent section.

\section{Tests on following path task}

The tests started on the indoor pool and they relied on moving the vehicle along the path specified by three way-points located in corners of the pool. The vehicle started at the right-down corner and moved successively to the right-upper, left-upper, and finally to left-down corner. In order to conduct the research on the pool, it was necessary to determine its orientation in Earth coordinate system which results from the fact that the CCC (Command Control Console) uses geographical coordinates to define a path, not coordinates in a local XY system. After determining the orientation of the pool, its length and width were measured and then also longitude and latitude of the corners were fixed. This way, location of the pool in an assumed geographical coordination system was determined. The path covered by the vehicle during the tests and defined in the CCC is presented in fig. 2 .

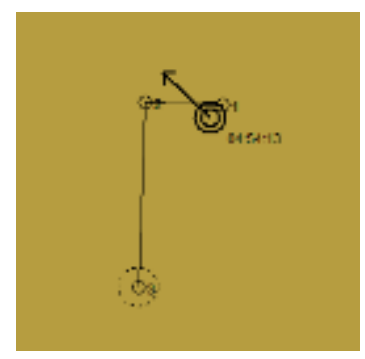

Fig. 2. Path of the vehicle during tests on the pool

First tests on the pool revealed unexpected behaviour of the vehicle which at two points of the pool was suddenly turning 30-40 degrees instead of proceeding to a next waypoint. As it turned out later, the reason was the influence of external magnetic field on compass OS5000 which, as mentioned above, is the main sensor used to determine the course. The disturbances of the magnetic field were, in turn, the result of mechanical infrastructure that was situated under the pool and which 
was the source of that field. Since the infrastructure mentioned above were necessary for proper operation of the hotel where the tests were performed, it could not be turned off and in consequence it accompanied the project team to the very end of the tests.

After determining reasons of unexpected vehicle manoeuvres, which as mentioned above, occurred only in two points of the pool, the experiments on the path following task were continued. They revealed that the NS 'shows' a wrong vehicle position even though the trajectory of the vehicle is correct. Fig. 3 presents the situation in which the vehicle is outside a desired path and simultaneously it is also outside the pool. In fact, the vehicle moved along edges of the pool moving from one waypoint to the other one.

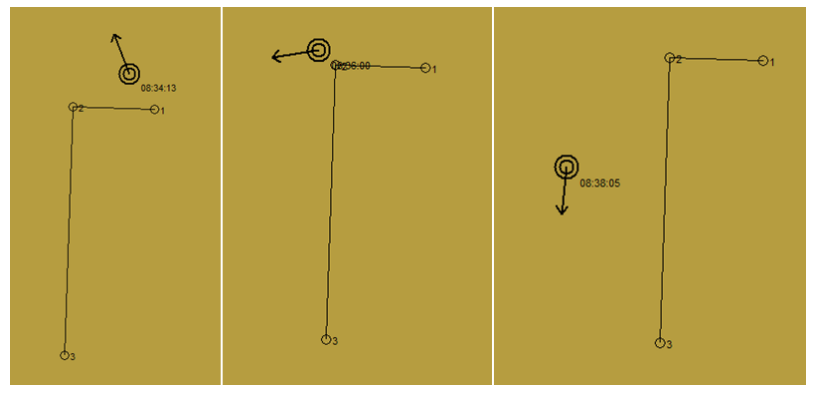

Fig. 3. Wrong indications of NS during moving the vehicle along a desired path:

the reason of the situation presented in the figure was wrong estimation of vehicle speed, the NS assumed higher speed and in effect the BAUV was always outside the pool, meanwhile, it moved with a smaller speed inside the pool

On the one hand, the cause of the above-mentioned situation was the software which recognizes a waypoint as reached if position of the vehicle is less than 10 meters away from it. This is why the NS 'saw' the vehicle outside the waypoints and the pool even though it correctly followed the path.

On the other hand, the cause of the wrong indications of the NS were also errors of dead reckoning which were the consequence of simplifications applied in calculations. To calculate position of the vehicle, the NS used one value of the average speed for each working mode. Meanwhile, the vehicle when moving between waypoints regulates the depth by means of lateral fins changing their neutral point. Each change, in turn, decreases progressive speed of the vehicle (see fig. 3). Moreover, in the calculations, the assumption was made that the vehicle moves with a constant velocity, meanwhile, it is of course not the case, each change of the working mode requires change of speed with an acceleration. 
All the above factors were considered in a next version of the NS which improved its accuracy. Example performance of the NS after improvements is depicted in fig. 4.

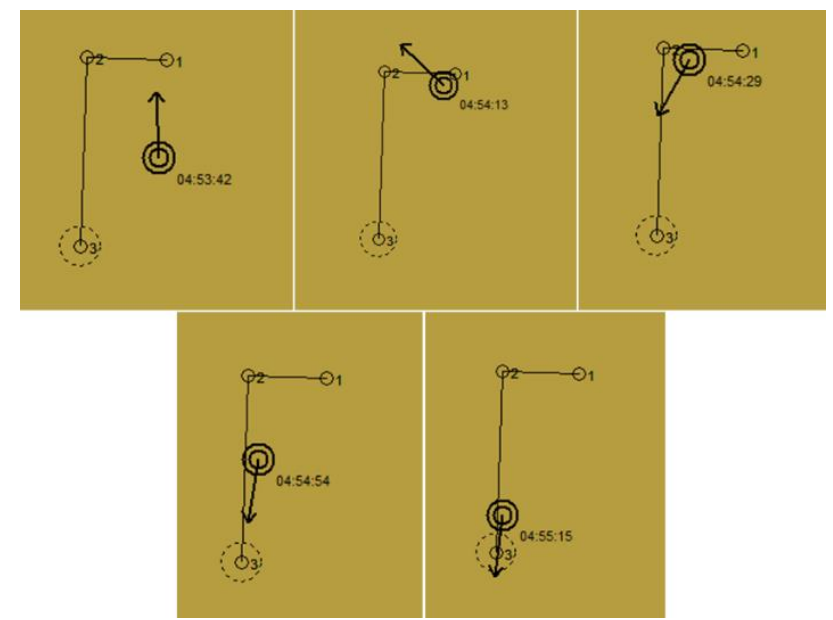

Fig. 4. Example operation of NS after improvements

A next stage of the research with the NS were tests on Osowskie lake in which the vehicle was moving either 40 degrees to a waypoint located in distance 100 meters away from a launching point, or along a triangle-shape-path presented in fig. 5.

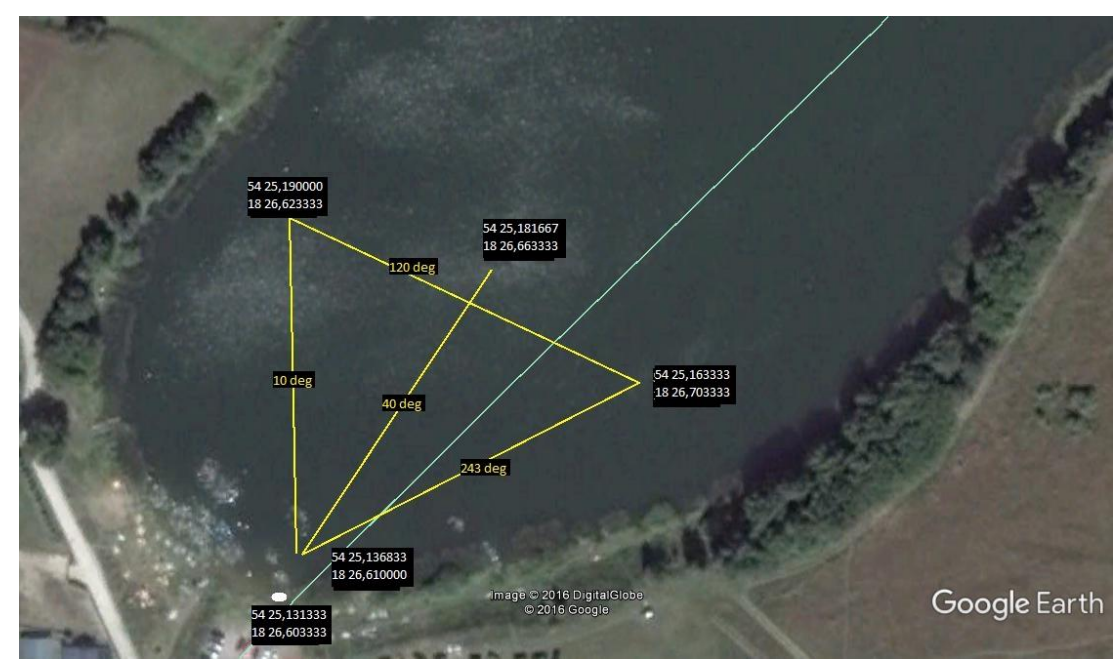

Fig. 5. Predefined path of the vehicle during tests on the Osowskie lake 
Generally, the tests revealed slight position errors caused by the wind and motion of water due to flowing nature of the lake. Since one objective of the research on the lake was to estimate vehicle errors (for example, errors resulting from asymmetry of construction, systematic errors of compass) which affect correctness of the dead reckoning and which should be determined in conditions without the influence of environmental factors, observed motion of water contributed to changing the conception of the research and moving them to the sea. The decision was made that estimation of the forces having influence on position errors should not be performed separately for each force but it should be done simultaneously for all the forces. That is, two estimations should be calculated, one for environmental factors and the other one for factors associated with the vehicle itself, however, they should be fixed when both factors together affect behaviour of the vehicle.

To this end, a special two-stage procedure was designed whose a single stage can be shortly described as follows: the vehicle moves underwater an assumed distance, for example 100 meters, $\mathrm{Ci}(\mathrm{i}=1,2)$ degrees, then, surfaces, records accurate GPS position, and calculates an error. The stages differ in $\mathrm{C}$ value which for the first stage ( $\mathrm{i}=1$ ) is equal to 45 degrees whereas for the second stage ( $\mathrm{i}=2$ ) 225 degrees. At the end of the procedure, position errors calculated for each stage are used to estimate both factors that according to the assumption cause the errors. In detail, the procedure is described in [3].

The procedure above was automated through adding its software implementation to the set of vehicle operations as a new operation which can be activated by the operator by means of the CCC. The other useful skill implemented in the vehicle after tests on the lake was ability to counteract the sea current. It enables the vehicle to move along a straight the shortest line to a waypoint under the influence of the sea current which pushes the vehicle out of the path.

Tests on the Gańsk Bay in the village Mechelinki were a next stage of the research verifying ability of the vehicle to follow a path. During the tests, the task of the vehicle was to follow different predefined paths with application of estimation procedure and counteracting the sea current outlined above. Example paths of the vehicle defined in the CCC are presented in fig. 6.

The research at sea revealed that accurate estimation of the sea current is very hard which may be due to significant changeability of the current at the testing area. Estimation procedure was run many times and each time the calculated direction of the current was different. Maximal differences in estimations for various trials amounted even to 180 degrees. The only sensible conclusion from that result seems to be impossibility to consider the current during the dead reckoning. 


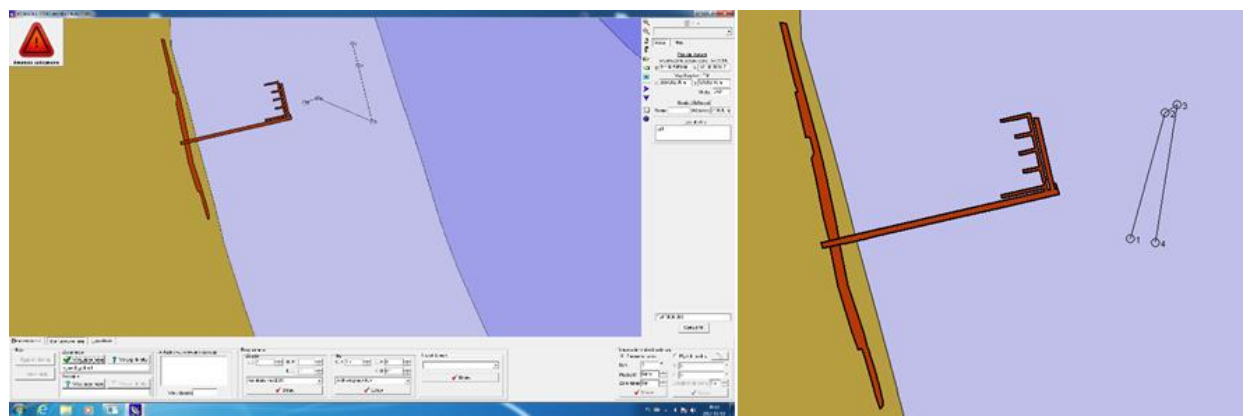

Fig. 6. Example paths during research in Mechelinki

In spite of the observed difficulties position errors of the dead reckoning were relatively small. Maximal error which appeared during the tests amounted to approximately 30 meters on the distance 100 meters.

\section{Tests on collision avoidance}

This part of the research began at the indoor pool and they initially relied on analysis of echo-sounder operation and data provided by them. During the tests, incidental wrong indications of the echo-sounders were noticed which in majority of cases happened for a small distance between an echo-sounder and an obstacle sensitivity of echo-sounders mounted in the vehicle amounts to $0.5 \mathrm{~m}$. In consequence, the software responsible for providing information about obstacles and distance to the sea bottom had to be insignificantly modified. Moreover, the software which gives high-level commands to the vehicle, e.g. turn left, go down $5 \mathrm{~m}$, was also improved to protect the vehicle from approaching the bottom at the distance lower than $0.5 \mathrm{~m}$.

In the next stage, ability to avoid collisions was tested. Due to the fact that operation of a front echo-sounder was severely disturbed at the pool (reflection of acoustic signal from walls of pool) which in practice made it impossible to apply it to detect obstacles, the decision was made to skip an obstacle detection phase in the whole collision avoidance process leaving only functionality responsible for the avoidance itself. To this end, a data base including obstacles which in normal conditions is fed with data from echo-sounders was filled with virtual obstacles located in the middle of the pool. A virtual wall composed of virtual obstacles was inserted to the data base (or simply to the environmental map) which forced the vehicle to turn towards one of the pool walls instead of moving straight to a way-point. In effect, a proper maneuvering of the vehicle was observed. The only abnormalities 
which were noticed related to position errors of the NS. Their occurrence made it impossible, in practice, to reliably evaluate behavior of the vehicle in conditions of obstacle presence.

In consequence, further tests focused on the collision avoidance were continued at open areas. They were performed in the village Mechelinki. Unfortunately, activation of the collision avoidance system caused chaotic behavior of the vehicle which manifested by continuous maneuvering. After analysis of logs from the vehicle it turned out that there are two main reasons of abnormal vehicle behavior. The first one was shallowness of the testing area and the other one was permanent trim of the vehicle with the 'head' lowered down. The consequence was that the front echo-sounder used to detect obstacles in front of the vehicle was detecting them all the time, it simply interpreted elements of the sea bottom as obstacles. In turn, the presence of the obstacles in the close proximity of the vehicle caused its unexpected chaotic behavior.

The other problem was occurrence of non-existing obstacles derived from acoustic signals reflected from the water surface. All the phenomenon outlined above forced the project team to impose modifications to the vehicle software. Unfortunately, they were not tested up to the point of preparing the paper.

\section{Tests on performing a mission}

The tests whose goal was to verify ability of the vehicle to follow a predefined path and simultaneously to handle cameras and sonar, emergency situations, and other occurring events were the last stage of experiments with the BAUV and they took place at sea in village Mechelinki. Example path along with definition of two waypoints (No. 1 and 2) is presented in fig. 7 and 8. Starting point of the mission was located in the southern corner of the promenade visible in the chart. Then, the vehicle was supposed to reach the waypoint No. 1 in which the underwater camera should be run. Once waypoint No. 2 is reached, the underwater camera should be turned off and the surface camera should be activated for a specified time and for vehicle course 225 degrees (the vehicle should maintain the course for a time of camera operation) which means that it should face the end of the promenade in Mechelinki. As it turned out, the mission outlined above, with and without GPS, was performed correctly. In addition to the mission above also other similar missions were also tested with the same satisfying effect.

Two problems were not solved in a satisfying way up to the very end of the tests. The first one was unsatisfactory accuracy of the NS during vehicle voyage. Unfortunately, the vehicle is not equipped with any speedometer whereas sensors for spatial 
orientation are characterized by errors which is the main cause of inaccuracies of underwater navigation which as mentioned above amounted maximally 30 meters on distance 100 meters.

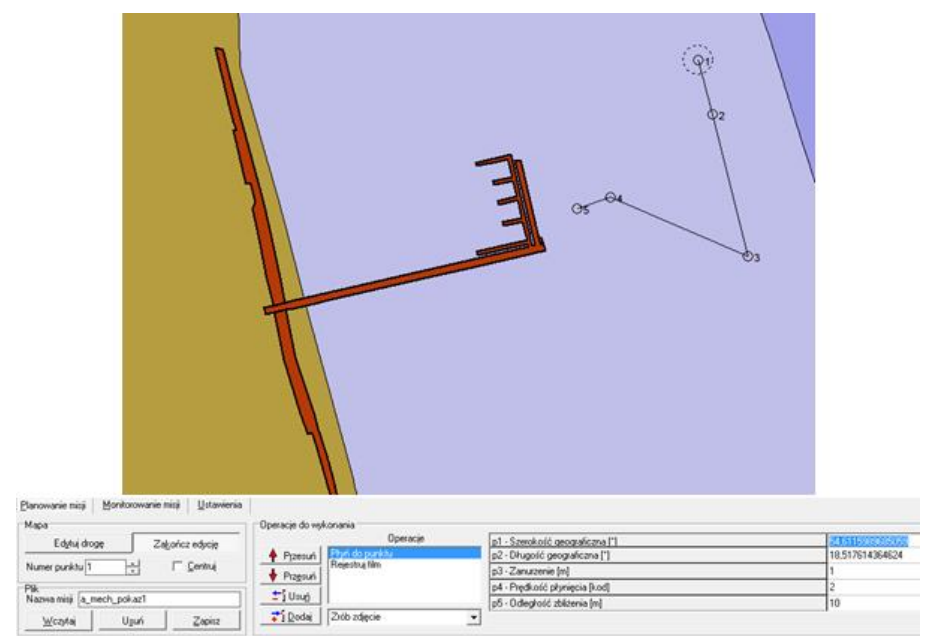

Fig. 7. Plan of example mission along with definition of waypoint No. 1

In order to improve precision of the navigation two solutions can be used. The first one is to equip the vehicle with a Doppler log as well as with an optical gyro, the first device measures velocity taking the sea current into account whereas the second one is considerably more accurate than compass OS5000 and INS-VN200 and what is more it is also robust to external magnetic field. Unfortunately, the drawback of the devices above is their price, weight and size. For small vehicles, working in a swarm - construction of such vehicles is ambition of the project team - it is not possible, for example, application of Doppler logs which are simply too large and too heavy.

The other solution for improvement accuracy of the dead reckoning is application of estimation methods that rely on Kalman filter (EKF, UKF) or Particle filter. To this end, more reliable mathematical model of the vehicle should be developed. The model used currently on the vehicle and reduced only to determine an average progressive speed without taking into account the influence of different parameters affecting behavior of the vehicle is definitely insufficient.

Another problem which was not solved up to the very end of experiments described in this paper was application of echo-sounders to detect obstacles and their false detections related to the sea bottom. The collision avoidance itself was tested in simulation and on the pool with satisfactory result, however, effective obstacle detection with the use of echo-sounders requires further works. 


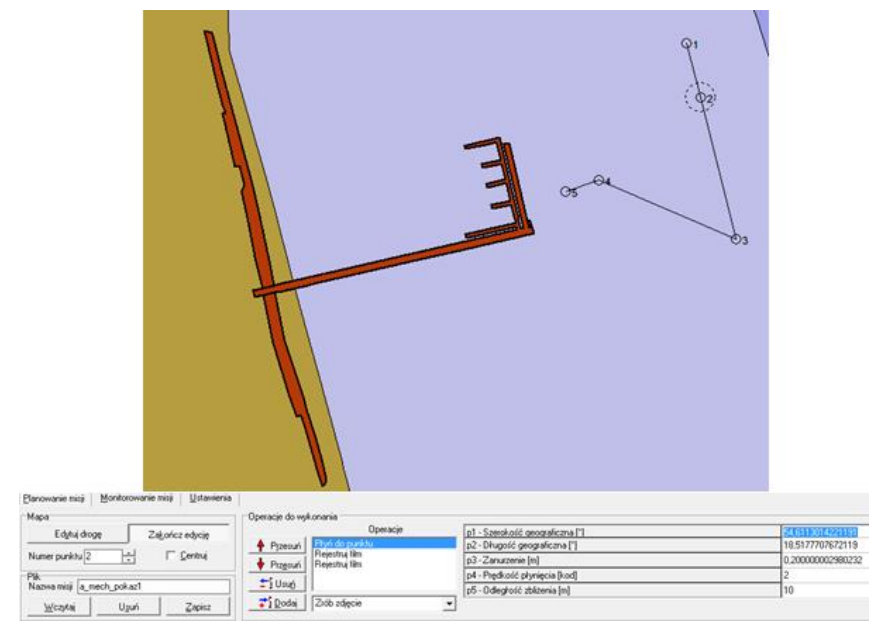

Fig. 8. Plan of example mission along with definition of waypoint No. 2

\section{CONCLUSIONS}

The paper constitutes the second part of the final report from the experiments carried out with the real biomimetic autonomous underwater vehicle designed within the project entitled 'Autonomous underwater vehicles with silent undulating propulsion for underwater reconnaissance' financed by National Center of Research and Development. The research described in this paper was focused on navigation system and autonomous operation. The experiments described in the report took place in a different locations and they lasted about six months with short breaks. As a result of all the tests, the vehicle has been designed which is capable of performing almost all predefined tasks and which meets massive majority of the assumptions made at the initial phase of the project. In short, the vehicle is able to perform simple commands in remotely operated mode, to autonomously follow a preprogrammed path with simultaneous operation on its sensors, to pass all recorded data by means of three communication channels, including underwater acoustic channel, and to handle different emergency situations.

In spite of the fact that the project as a whole as well as the research reported in the paper are undeniable success because they produced first in Poland fully equipped (not a toy) autonomous biomimetic underwater vehicle, further research are necessary in two areas. The first one is collision avoidance in shallow waters and the second one is accurate underwater navigation based on the odometry 
and low-cost navigational sensors. Both areas were 'touched' during the project, however, the results which were achieved do not satisfy the project team and generally they require improvement within further works.

\section{Acknowledgements}

The development and testing of the BAUV was founded by Polish National Center of Research and Development under project No. DOBR-BIO4/033/13015/2013, entitled 'Autonomous underwater vehicles with silent undulating propulsion for underwater reconnaissance'.

\section{REFERENCES}

[1] Malec M., Morawski M., Zając J., Fish-like swimming prototype of mobile underwater robot, 'Journal of Automation. Mobile Robotics \& Intelligent Systems', 2010, Vol. 4, No. 3, pp. 25-30.

[2] Praczyk T., A quick algorithm for planning a path for biomimetic autonomous underwater vehicle, 'Scientific Journals of Maritime University of Szczecin', 2016, No. 45, pp. 23-28.

[3] Praczyk T., Correction of navigational information provided for biomimetic autonomous underwater vehicle, 'Polish Maritime Research', in press.

[4] Praczyk T., Szymak P., Software architecture of biomimetic underwater vehicle, Proc. SPIE 9831, Ground/Air Multisensor Interoperability, Integration, and Networking for Persistent ISR VII, 2016, DOI:10.1117/12.2225871.

[5] Praczyk T., The influence of parameters of biomimetic underwater vehicle control system on the ability of the vehicle to avoid obstacles, 'Scientific Journal of Polish Naval Academy', 2016, Vol. 205, No. 2, pp. 75-91.

[6] Praczyk T., Using Genetic Algorithms for Optimizing Algorithmic Control System of Biomimetic Underwater Vehicle, 'Computational Methods in Science and Technology', 2015, Vol. 21, No. 4, pp. 251-260.

[7] Szymak P., M. Malec, M. Morawski, Directions of development of underwater vehicle with undulating propulsion, 'Polish Journal of Environmental Studies', 2010, Vol. 19, No. 3, pp. 107-110.

[8] Szymak P., Praczyk T., Naus K., Malec M., Morawski M., Research on biomimetic underwater vehicles for underwater ISR, Proc. of SPIE 9831, Ground/Air Multisensor Interoperability, Integration, and Networking for Persistent ISR VII, 2016, DOI:10.1117/12.2225587.

[9] CyberFish, [online], http://www.cyberryba.pl/ [access 05.11.2017]. 


\section{RAPORT Z BADAŃ NAD BIOMIMETYCZNYM AUTONOMICZNYM POJAZDEM PODWODNYM NAWIGACJA I AUTONOMICZNY SYSTEM STEROW A I A}

\section{STRESZCZENIE}

W artykule przedstawiono część drugą końcowego raportu z prac zrealizowanych w ramach projektu „Autonomiczne pojazdy podwodne z cichym napędem falowym” współfinansowanego przez Narodowe Centrum Badań rozwoju w latach 2013-2017.

Słowa kluczowe:

autonomiczny pojazd podwodny, biomimetyczny autonomiczny pojazd podwodny, regulacja kursu i głębokości, sterowanie autonomiczne.

Article history

Received: 25.01 .2018

Reviewed: 04.03.2018

Revised: 26.04.2018

Accepted: 27.04.2018 